\title{
Nursing Care of Low-Risk Newborns in Low Resource Setting: Nurses' Aides May Bridge the Gap at Community Hospitals
}

\author{
Subhashchandra Daga
}

\section{ABSTRACT}

Objective: To study the role of a nurses' aide in the care for newborns weighing between 1500 and $2000 \mathrm{~g}$ at birth in a low resource setting.

Study Design: Observational.

Setting: The General hospital in $\mathbf{1 9 9 4 - 9 5}$, in a public sector, located in a remote area in India

Intervention: A female ward assistant with seven years of schooling trained, on-thejob, to keep babies warm, initiate maternal breastfeeding, and to detect rapid breathing. The nursing staff from the pediatric ward supervised her performance. A separate "warm room" appropriately heated for preterm and sick babies became a makeshift nursery. The nursing staff administered enteral feeding, oxygen, and antibiotics. Services of the resident doctors or general duty medical officers were not available.

Results: The survival rate was nearly $100 \%$ for babies with birthweights between 1,500 and 2,000 $\mathrm{g}$ (none referred out).

Conclusions: A nurses' aide may facilitate the delivery of special care for newborns where nursing personnel are grossly inadequate and saving babies weighing between 1,500 and $2,000 \mathrm{~g}$ may need minimal inputs. It may be worthwhile to target 1,500 and $2,000 \mathrm{~g}$ birthweight categories even when resources are meager.

What is already known about this subject?

- Low resource settings face staff shortages, especially nursing staff.

- Health workers with midwifery skills can deliver nearly $90 \%$ of essential care services for maternal and neonatal health.

- A substantial proportion of neonatal deaths occur among moderately low birth weight babies.

What does this study add?

- It is possible to train a semi-literate person to facilitate early breastfeeding and to keep a baby warm.

- A large proportion of deaths among babies with birthweight ranging from 1500 to $2000 \mathrm{~g}$ are preventable with meager resources.

How might this impact on clinical practice or future developments?

The facilities facing shortage of nursing staff in low resource settings, may employ nurses' aide to deliver basic newborn care.

Keywords: Low birth weight, neonatal care in low resource setting, neonatal mortality, nurses' aide.

Published Online: January 4, 2022

ISSN: $2736-5476$

DOI: $10.24018 /$ ejclinicmed.2022.3.1.158

S. Daga*

Emeritus Prof. B.J. Medical College and

Sassoon General Hospital, India.

(e-mail: dagasubhash49@gmail.com)

\section{INTRODUCTION}

In 2015, nations across the world began working toward a new global development agenda seeking to achieve targets established by the Sustainable Development Goals (SDG) for 2030. By that time, the proposed SDG targets for child mortality stipulate that neonatal mortality rates should be lower, to a maximum of 12 deaths per 1,000 live births. In comparison, under-five mortality rates should be less than 25 deaths per 1,000 live births [1]. More countries may miss the SDG targets on neonatal mortality rates than those missing the targets related to under-five mortality if current trends continue. More than 60 countries are likely to miss the 2030 targets for neonatal mortality rates, while half may not reach these targets until after 2050 [1].

The study center, attached to a medical school, lacked optimal infrastructure, adequate staff, and even essential neonatal care. Many trained staff nurses are also unwilling to accept jobs at this center, partially because of its remote location. Indeed, approximately $35-40 \%$ of these posts remain vacant. Similar situations exist in other developing countries such as Ghana and Kenya [2], [3], where many crucial components are missing, including essential neonatal care equipment, drugs, and personnel trained to perform 
crucial life-saving actions.

This study reviewed the situations and interventions nearly two decades prior. The conditions at Indian medical colleges have vastly improved since the Medical Council of India implemented strict regulations. However, this study's results may be relevant for first-referral units providing newborn care in settings with meager resources. Most importantly, a nurse's aide may be a catalyst in extending such services even at a primary health center, where the nurses are busy with the field activities, in case the parents express an inability to access referral services.

\section{PAtIENTS AND MEthodS}

The hospital, now attached to a medical school in India, once functioned as a district hospital. The study was conducted during 1994 and 1995. The hospital did not contain a neonatal nursery. Thus, sick babies and those weighing less than $2 \mathrm{~kg}$ were referred to private care facilities since the nursing staff was grossly inadequate in number. In a pediatric ward containing 30 beds, either two staff nurses or one staff nurse and nurse-in-charge were present during the morning shift, while one staff nurse was available for each of the afternoon and night shifts. Three consultants, two full-time and one part-time, were available. However, no resident doctors or general duty medical officers were present to assist these consultants.

This study reviewed the situations and interventions nearly two decades prior. The conditions at Indian medical colleges have vastly improved since the Medical Council of India implemented strict regulations. However, this study's results may be relevant for first-referral units providing newborn care in settings with meager resources. Most importantly, a nurse's aide may be a catalyst in extending such services even at a primary health center, where the nurses are busy with the field activities, in case the parents express an inability to access referral services.

The target population for this study was babies weighing between 1,500 and $2,000 \mathrm{~g}$. Services of a minimum number of nurses were utilized. The prime interventions were provisions of adequate warmth and proper feeding. A12 X $10 \mathrm{ft}$ draft-free storeroom in the pediatric ward had been converted into a warming room for this purpose. The temperature in this area was maintained at around $25^{\circ} \mathrm{C}$ using an electric room warmer that cost approximately INR 300 (4 USD) [4]. A baby weighing less than 1,500 $\mathrm{g}$ was placed in a Styrofoam box [5] in the warm room. Babies were adequately clothed. Warm foot-soles indicated thermal adequacy, but body temperatures were not recorded [6]. Babies were breastfed, spoon-fed, or fed by orogastric tubes based on birth weight, postnatal age, and respiratory status. For the first two to three days, babies were fed a $5 \%$ dextrose solution in addition to milk from human donors or the mother (including colostrum). Babies with respiratory distress also received oxygen and antibiotics. Ampicillin and gentamicin were administered IM at $50 \mathrm{mg} / \mathrm{kg} / \mathrm{dose}$ twice daily and $6 \mathrm{mg} / \mathrm{kg} \mathrm{IM}$ doses once daily, respectively. Oxygen was administered through an intranasal catheter at 0.5-1 liter/min from a 660-liter portable cylinder since piped oxygen was not available. No investigations were performed, and no intravenous medications were administered in almost every situation. Portable X-ray services were not available.

A nurse's aide with seven years of schooling, already working as a ward assistant, was informally trained to keep babies warm, help mothers initiate breastfeeding, and detect rapid breathing. The hands-on training, conducted on ward rounds included the examination of foot soles for the adequacy of warmth and helping the mother put the baby to breast or express breast milk. She aided in the spoon/breastfeeding process, often with the help of mother/mother-in-law. She was repeatedly shown babies with normal breathing and those with rapid breathing. She also shared non-nursing responsibilities. Her work hours were from $7 \mathrm{AM}$ to $3 \mathrm{PM}$, five days per week. The nursing staff shared the responsibilities when the nurse aide was not on duty. She received training on-the-job conducted by a consultant (SD). The nurse's aide performed duties under the supervision of staff nurses from the pediatric ward. An onstaff nurse administered enteral feeding, oxygen, and antibiotics. The record-keeping was minimal. However, simple guidelines that covered feeding and antibiotic dosages were available for the staff nurses.

Labor room interventions included the provision of the following items: A room heater to keep babies warm, a bag and mask for the resuscitation of asphyxiated babies, a suction bulb to apply oropharyngeal suction in cases involving meconium-stained amniotic fluid, and a Styrofoam box to transport babies from the labor room to the makeshift nursery. The labor room was relatively better staffed, with at least two staff nurses on duty at all times.

\section{RESULTS}

\section{A. Inborn Babies}

$\mathrm{f}$ the 38 inborn babies weighing between 1,500 and 2,000 $\mathrm{g}$ at birth, 37 (97.6\%) survived, and one was discharged against medical advice (DAMA) in stable condition. Of the 23 babies admitted with birth weights between 1,000 and $1,500 \mathrm{~g}, 16(69.3 \%)$ survived, and two (8.6\%) died. Five babies were DAMA, three of whom were in an unstable condition. Among the five babies weighing less than 1,000 $\mathrm{g}$, four died, and one was DAMA in stable condition (Table I).

TABLE I: BIRTHWEIGHT DISTRIBUTIONS AND OUTCOMES AMONG INBORN BABIES (1994-95)

\begin{tabular}{cccc}
\hline \hline $\begin{array}{c}\text { Birth Weight } \\
(\mathrm{Kg})\end{array}$ & Survived (No) & DAMA* (No) & Died (No) \\
\hline \hline Less than 1 & - & $1(20 \%)$ & $4(80 \%)$ \\
$1-1.5$ & $16(69.3 \%)$ & $5(22.1 \%)(3)$ & $2(8.6 \%)$ \\
$1.55-2$ & $37(97.6 \%)$ & $1(2.4 \%)(0)$ & - \\
$2.05-2.5$ & $7(70 \%)$ & $2(20 \%)(0)$ & $1(10 \%)$ \\
More than 2.5 & 4 & - & - \\
\hline \hline
\end{tabular}

\section{B. Out-born Babies}

Of the 12 babies admitted with birth weights between 1,500 and 2,000 g, eight were DAMA (seven of these were in stable condition). Two were discharged, and two died. Eleven babies were admitted with birth weights between 1,000 and $1,500 \mathrm{~g}$. Of these, six (54.4\%) were DAMA (five in stable condition). Three (27.2\%) were discharged, and 
two $(18.4 \%)$ died (Table II). DAMA rates were higher among out-born babies. No baby required a second round of antibiotics.

TABLE II: BIRTHWEIGHT DISTRIBUTIONS AND OUTCOMES AMONG OUTBORN BABIES (1994-95)

\begin{tabular}{cccc}
\hline \hline $\begin{array}{c}\text { Birth weight } \\
(\mathrm{Kg})\end{array}$ & Survived (No) & DAMA* (No) & Died (No) \\
\hline \hline Less than 1 & - & 1 & - \\
$1-1.5$ & $3(27.2 \%)$ & $6(54.4 \%)(1)$ & $2(18.4 \%)$ \\
$1.55-2$ & $2(16.6 \%)$ & $8(66.8 \%)(1)$ & $2(16.6 \%)$ \\
$2.05-2.5$ & $3(60 \%)$ & $1(20 \%)(1)$ & $1(20 \%)$ \\
More than 2.5 & $3(75 \%)$ & $1(25 \%)(1)$ & - \\
\hline \hline
\end{tabular}

Numbers in second parentheses indicate unstable babies at time of discharge.

*Discharged against medical advice.

\section{DisCUSSION}

Essential structural components are required to provide special care for newborns, including adequately equipped facilities, appropriately trained healthcare professionals in adequate numbers, and life-saving equipment. We preferred "conservative" care to "conventional" neonatal care in the absence of minimum necessary nursing staff, capacity in particular, even for pediatric services [7]. This study reviewed the situations and interventions nearly two decades prior. The conditions at Indian medical colleges have vastly improved since the Medical Council of India implemented strict regulations. However, this study's results may be relevant for first-referral units providing newborn care in settings with meager resources. Most importantly, a nurse's aide may be a catalyst in extending such services even at a primary health center, where the nurses are busy with the field activities, in case the parents express an inability to access referral services.

The nurse's aide was employed since there was an insufficient number of nursing personnel. Her primary responsibilities involved keeping babies warm and facilitating early breastfeeding. Nursing staff administered enteral feeding, oxygen, and antibiotics. The nurse's aide also appears to have catalyzed the establishment of neonatal care services. The motivation for the study comes from an earlier experience of working in a rural community with insufficient capacity and/or capability of the nursing staff to provide the care required to care for the LBW and VLBW newborn babies and neonates [8]. Based on our earlier experience [8], we aimed to achieve a minimum $75 \%$ survival rate in the target population. However, we met a much higher percentage (nearly 100\%). Further, the $69.3 \%$ survival rate among babies weighing between 1,000 and $1,500 \mathrm{~g}$ was completely unexpected, as was the number of referrals coming from other hospitals.

The proportion of DAMA neonates was tiny in the target population, a proxy for parent satisfaction. Most DAMA neonates among out-born babies were due to reluctance to admit mothers to the general hospital. This trend was common among those who had received $\mathrm{C}$-sections at private nursing homes. No sooner, a baby accepted feeds, a request for discharge came in. The parents of only stable babies requested for DAMA.

A before-and-after comparison was not possible since, the LBW babies were referred to private facilities in absence of any form of care in the hospital. Those who could not afford private care, sooner or later, went against medical advice. No records were maintained.

Substantial progress has been made in child survival rates throughout the world since 1990. The global number of neonatal deaths declined from 5.0 million in 1990 to 2.5 million in 2017. However, the decline in neonatal mortality rates over this same period has been slower than those of post-neonatal under-five mortality rates $(51 \%$ and $62 \%$, respectively) [9]. The primary strategies used to mitigate neonatal mortality rates include services for small and sick newborns [9]. A study conducted in five low and one middle-income country revealed that $37 \%$ of neonatal deaths occurred among babies with birth weights between 1,500 and $2499 \mathrm{~g}$, while $21 \%$ of all deaths occurred in the 33-36week gestational age group [10]. Babies in this group require urgent attention. They are more likely to require short-term supervised care when compared to those with birth weights less than $1,500 \mathrm{~g}$, who typically need more specialized and expensive newborn intensive care over more extended periods. Available interventions can reduce neonatal deaths due to preterm, intrapartum, and infectionrelated deaths by $58 \%, 79 \%$, and $84 \%$, respectively [11]. Here, breastfeeding support and thermal protection are some of the interventions known to work [11]. These measures are doable with relative ease. It is also important to maintain normothermia immediately upon the birth of a preterm baby [12]. Maintenance of the "warm chain" for newborn care must, therefore, begin at the place of birth itself (i.e., the labor room) [13].

Maintaining a temperature of more than $25^{\circ} \mathrm{C}$ in the baby corner of the labor room was one of the primary interventions used in this study, so that body temperature upon admission is adequate. There is a documented association between hypothermia at Neonatal Intensive Care Unit admission and neonatal mortality [14]. Electric room heaters and Styrofoam boxes are reasonable options when radiant warmers/incubators are not feasible. Electrical room warmers are particularly sustainable interventions since they are repairable locally. On the other hand, individual warming devices (e.g., radiant warmers/incubators) are challenging to maintain in remote locations. Another critical child survival strategy is the promotion of breastfeeding. Here, early initiation has the potential to prevent $16 \%$ of neonatal deaths, when breastfeeding starts within the first hour of birth [15]. Health workers with midwifery skills can deliver nearly $90 \%$ of essential care services for maternal and neonatal health [16]. However, it is challenging to provide nursing staff in adequate numbers and with required skills for the management of neonatal services. Fewer than one in six high-burden countries currently meet the minimum threshold of 23 doctors, midwives, and nurses per 10,000 of the population requiring healthcare provisions [17].

In sum, even with meager resources, neonatal survival can be enhanced at community hospitals. The problem of an inadequate number of nursing staff can be addressed partly by the nursing aides. First, the efforts should concentrate on babies with birth weights between 1,500 and 2,000 g by providing warmth and initiating breastfeeding at the earliest. Nurse's aides can give support in instances of an inadequate 
number of nursing staff at a first referral unit and even at a primary health center.

\section{REFERENCES}

[1] UNICEF. Child survival and the SDGs. [Internet]. 2017. [cited 2019 Mar 28]. Available from: https://data.unicef.org/topic/childsurvival/child-survival-sdgs/.

[2] Vesel L, Manu A, Lohela TJ, Gabrysch S, Okyere E, ten Asbroek AHA, et al. Quality of newborn care: a health facility assessment in rural Ghana using survey, vignette and surveillance data. BMJOpen. 2013; 3: e002326.

[3] Opondo C, Ntoburi S, Wagai J, Wafula J, Wasunna A, Were F, et al. Are hospitals prepared to support newborn survival? - An evaluation of eight first-referral level hospitals in Kenya. Trop Med Int Health. 2009; 14(10): 1165-1172.

[4] Daga SR, Chandrashekhar L, Pol PP, Patole S. Appropriate technology in keeping babies warm in India.Ann Trop Paediatr. 1986; 6: 23-25.

[5] Daga SR. Styropor box for maintaining the warm chain in newborn care. Int Child Health. 1997; 8: 69-73.

[6] Daga SR. Simplified monitoring of sick newborns. Trop Doctor. 1998; 28: 228-32.

[7] Daga S, Daga A. Neonatal intensive care in the developing countries: conservative or aggressive approach? Child Health in the Tropics: Sixth Nutricia - Cow \& Gate Symposium Leuven, 18-21 October 1983. Springer: Dordrecht, 1983: 233-245.

[8] Borulkar PD, Borulkar SP, Dhole RK, Daga SR. Special care for newborns at a community hospital: a 5-year experience. Trop Doctor. 1998; 28: 201-203.

[9] World Health Organization. Newborns: reducing mortality. [Internet]. 2018. [cited 2019 Mar 28]. Available from: https://www.who.int/news-room/fact-sheets/detail/newbornsreducing-mortality.

[10] Belizán JM, McClure EM, Goudar SS, Pasha O, Fabian EF, Patel A, et al. Neonatal death in low- to middle-income countries: a global network study. Am J Perinatol, 2012; 29(08): 649-656.

[11] Akseer N, Lawn JE, Keenan W, Konstantopoulos A, Cooper P, Ismail Z. Ending preventable newborn deaths in a generation. Int J Gynecol Obstet. 2015; 131: S43-S48.

[12] Almeida MFB, Ginsberg R, Sancho GA, Rosa IRM, Lamy ZC, Martinez FE, et al. Hypothermia and early neonatal mortality in preterm infants. J Pediatr. 2014; 164: 271-275.

[13] World Health Organization. Thermal Protection of the Newborn: A Practical Guide. World Health Organization: Geneva, 1997.

[14] Liu Y, Shah PS, Ye XY, Warre R, Piedboeuf B,Deshpandey A, et al. Association between admission temperature and mortality and major morbidity in preterm infants born at fewer than 33 weeks' gestation. JAMA Pediatr. 2015; 169(4): e150277.

[15] Edmond KM, Zandoh C, Quigley MA, Amenga-EtegoS, OwusuAgyei S, Kirkwood BR. Delayed breastfeeding initiation increases risk of neonatal mortality. Pediatrics. 2006; 117: e380-e386.

[16] UNFPA. The State of the World's Midwifery 2014.UNFPA: New York, 2014.

[17] UNFPA, ICM, and WHO. State of the World's Midwifery 2011: Delivering Health, Saving Lives. UNFPA: New York, 2011. 